\title{
NEWTON-MULTIGRID FEM SOLVER FOR THE SIMULATION OF QUASI-NEWTONIAN MODELING OF THIXOTROPIC FLOWS
}

\author{
A. Ouazzi, N. Begum and S. Turek \\ Institute for Applied Mathematics, LS III, TU Dortmund University, D-44227 Dortmund, Germany \\ Abderrahim.Ouazzi@math.tu-dortmund.de \\ Naheed.Begum@math.tu-dortmund.de \\ ture@featflow.de (S. Turek)
}

Key words: Thixotropic Flows, Quasi-Newtonian Approach, FEM, Newton-Multigrid

\begin{abstract}
This paper is concerned with the application of Finite Element Methods (FEM) and NewtonMultigrid solvers to simulate thixotropic flows using quasi-Newtonian modeling.

The thixotropy phenomena are introduced to yield stress material by taking into consideration the internal material microstructure using a structure parameter. Firstly, the viscoplastic stress is modified to include the thixotropy throughout the structure parameter. Secondly, an evolution equation for the structure parameter is introduced to induce the time-dependent process of competition between the destruction (breakdown) and the construction (buildup) inhabited in the material. This is done simply by introducing a structure-parameter-dependent viscosity into the rheological model for yield stress material. The nonlinearity, related to the dependency of the diffusive term on the material parameters, is treated with generalized Newton's method w.r.t. the Jacobian's singularities having a global convergence property. The linearized systems inside the outer Newton loops are solved using the geometrical multigrid with a Vanka-like linear smoother taking into account a stable FEM approximation pair for velocity and pressure with discontinuous pressure and biquadratic velocity spaces.

We analyze the application of using the quasi-Newtonian modeling approach for thixotropic flows, and the accuracy, robustness and efficiency of the Newton-Multigrid FEM solver throughout the solution of the thixotropic flows using manufactured solutions in a channel and the prototypical configuration of thixotropic flows in Couette device.
\end{abstract}

\section{Introduction}

The thixotropic flows are based on viscoplastic constitutive law, namely Bingham model

$$
\left\{\begin{aligned}
\sigma=2 \eta \mathbf{D}(u)+\tau \frac{\mathbf{D}(u)}{\|\mathbf{D}(u)\|}, & \text { if }\|\mathbf{D}(u)\| \neq 0 \\
\|\boldsymbol{\sigma}\| \leq \tau, & \text { if }\|\mathbf{D}(u)\|=0
\end{aligned}\right.
$$

where $\mathbf{D}(u)$ denotes the strain rate tensor. The norm for a tensor $\Lambda$ is given by $\|\Lambda\|=\sqrt{\operatorname{Tr}\left(\Lambda^{2}\right)}$. We use $\|\mathbf{D}(u)\|$ and $\|\mathbf{D}\|$ alternately. $\eta$ denotes plastic viscosity, and $\tau$ defines a yield stress that is a threshold parameter from which the material start yielding. The shear stress has two contributions: a viscous part, and a strain rate independent part. Thixotropic phenomena, as a competition of two processes, build up and breakdown, are introduced into rheological model for yield stress material through a structure- 
parameter. A simple linear/affine function for plastic viscosity and yield stress w.r.t. structure parameter are adopted i.e. Houška's thixotropic model [3],

$$
\left\{\begin{array}{l}
\eta(\lambda)=\eta_{0}+\lambda \eta_{1} \\
\tau(\lambda)=\tau_{0}+\lambda \tau_{1}
\end{array}\right.
$$

where $\eta_{0}$ and $\tau_{0}$ are the initial plastic viscosity and yield stress, respectively, in the absence of any thixotropic phenomena. $\eta_{1}$ and $\tau_{1}$ represent the rates of changes of plastic viscosity and yield stress, respectively, w.r.t. structure parameter. The time-dependent process of competition between the destruction (breakdown) and the construction (buildup) inhabited in the material is given by the following structure-parameter equation

$$
\left(\frac{\partial}{\partial t}+u \cdot \nabla\right) \lambda-a(1-\lambda)-b\|\mathbf{D}\| \lambda=0
$$

where, $a$ and $b$ are dimensionless rate constants for creation (build up) and breakage (breakdown).

The quasi-Newtonian modeling approach for thixotropic flows is based on a viscosity approach for yield stress flows in which an extended viscosity, well-defined on all domain, is introduced $\eta(\cdot, \cdot)$ dependent on the strain rate and the structure parameter. Indeed, let us introduce the second invariant of the strain rate tensor $D_{\mathbb{I}}=\frac{1}{2}(2 \mathbf{D}(u): 2 \mathbf{D}(u))$, and define the regularized viscosity, as for instance [8]

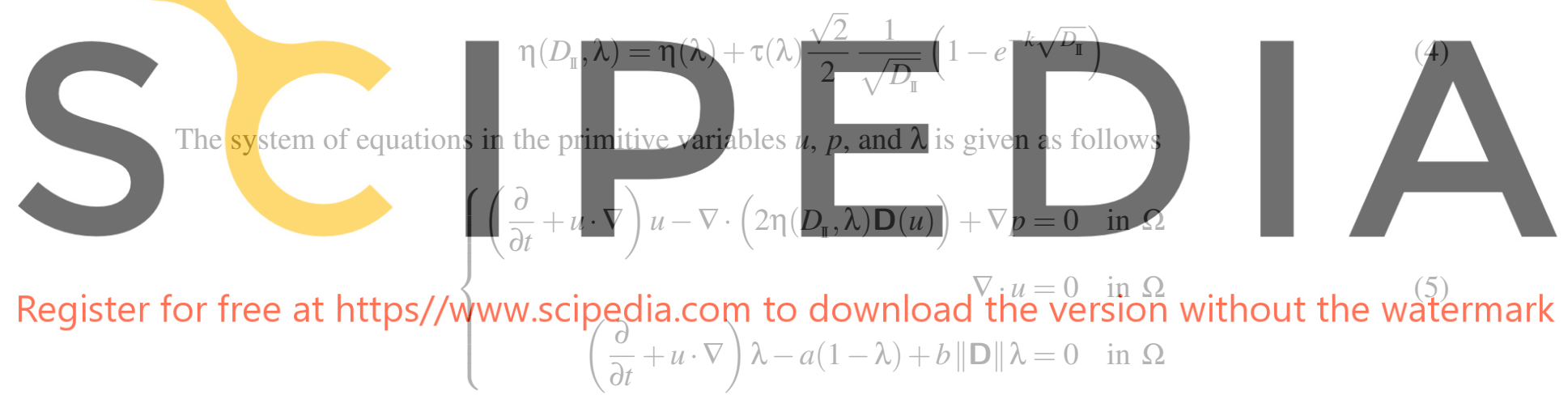

The viscous term in (5) exhibits infinitely high viscosity in the limit of low/vanishing shear rates. Additionally, the accuracy of the solution is strongly dependent on $k$. In order to obtain an accurate solution, the value of $k$ needs to be very high which deteriorates the solver on the other hand.

\section{Finite element discretization}

To derive the variational form for thixotropic flows, we consider the spaces $\mathbb{T}:=L^{2}(\Omega), \mathbb{V}:=\left(H_{0}^{2}(\Omega)\right)^{2}$, and $\mathbb{Q}:=L_{0}^{2}(\Omega)$ associated, respectively, with the corresponding $L^{2}$-norm, $\|\cdot\|_{0}, H^{1}$-norm, $\|\cdot\|_{1}$, and $L^{2}$ norm, $\|\cdot\|_{0}$. Let $\tilde{u}:=(\lambda, u, p) \in \mathbb{T} \times \mathbb{V} \times \mathbb{Q}$, and $\tilde{v}:=(\xi, v, q) \in \mathbb{T} \times \mathbb{V} \times \mathbb{Q}$ a test function. The weak formulation for the thixotropic flows reads: Find $\tilde{u} \in \mathbb{T} \times \mathbb{V} \times \mathbb{Q}$ s. $t$.

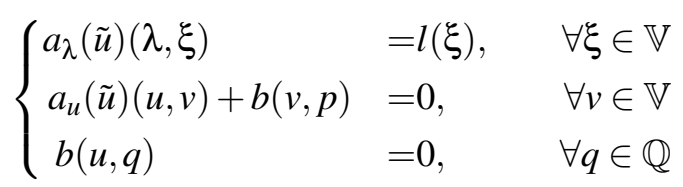


Or, equivalently: Find $\tilde{u} \in \mathbb{T} \times \mathbb{V} \times \mathbb{Q}$ s. $t$.

$$
a_{\lambda}(\tilde{u})(\lambda, \xi)+a_{u}(\tilde{u})(u, v)+b(v, p)-b(u, q)=l(\xi, v, q) \quad \forall \tilde{v} \in \mathbb{T} \times \mathbb{V} \times \mathbb{Q},
$$

where $a_{\lambda}(\tilde{u})(\cdot, \cdot), a_{u}(\tilde{u})(\cdot, \cdot), b(\cdot, \cdot)$, and $l(\xi, v, q)$ are given as follows

$$
\begin{gathered}
a_{\lambda}(\tilde{u})(\lambda, \xi)=\int_{\Omega}(a+b\|\mathbf{D}\|) \lambda \xi d \Omega+\int_{\Omega} u \cdot \nabla \lambda \xi d \Omega, \\
a_{u}(\tilde{u})(u, v)=\int_{\Omega} 2 \eta\left(D_{\mathbb{I}}, \lambda\right) \mathbf{D}(u): \mathbf{D}(v) d \Omega+\int_{\Omega} u \cdot \nabla u v d \Omega, \\
b(v, q)=-\int_{\Omega} \nabla \cdot v q d \Omega, \\
l(\xi, v, q)=l(\xi)=\int_{\Omega} a \xi d \Omega .
\end{gathered}
$$

The finite element approximations of the problem (7) have to take care of its saddle point character, due to the bilinear form (10). Furthermore, since thixotropic flows are slow, the only remaining issue is the control/continuity of the bilinear form (8) in the norm of space $\mathbb{T}$.

We opt for higher order stable pair biquadratic for velocity and piecewise linear discontinuous for the pressure, $Q_{2} / P_{1}^{\text {disc }}$, and higher order quadratic for structure parameter $Q_{2}$ with the appropriate stabilization for the convective terms $[6,7]$. Indeed, let the domain $\Omega$ be partitioned by a grid $K \in \mathcal{T}_{h}$ which are assumed to be open quadrilaterals such that $\Omega=\operatorname{int}\left(\bigcup_{k \in \mathcal{T}_{h}} \bar{K}\right)$. For an element $K \in \mathcal{T}_{h}$, we denote by $\mathcal{E}(K)$ the set of all 1-dimensional edges of $K$. Let $\mathcal{E}_{i}:=\bigcup_{k \in \mathcal{T}_{h}} \mathcal{E}(K)$ be the set of all interior element edges of the grid $\mathcal{T}_{h}$.

We define the conforming
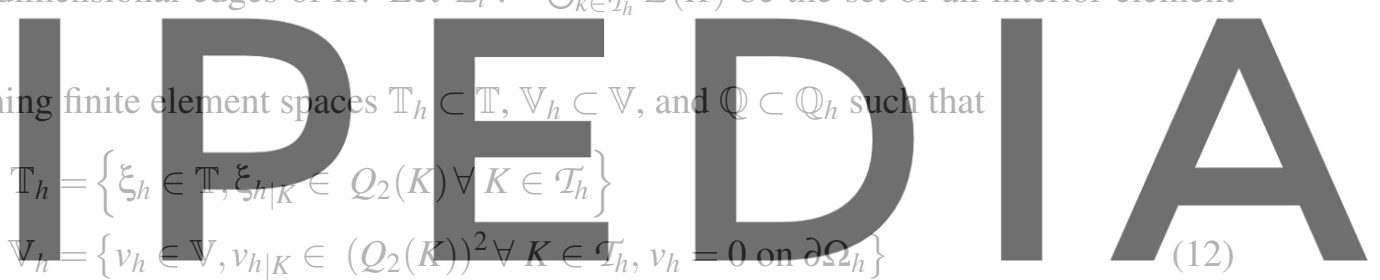

Register for free at https//whw.sciped fal.com to downthad the version without the watermark

The approximate problem reads: Find $\tilde{u} \in \mathbb{T}_{h} \times \mathbb{V}_{h} \times \mathbb{Q}_{h} s . t$.

$$
\left\{\begin{aligned}
a_{\lambda}(\tilde{u})(\lambda, \xi)+j_{\lambda}(\lambda, \xi) & =l(\xi), & & \forall \xi \in \mathbb{T}_{h} \\
a_{u}(\tilde{u})(u, v)+b(v, p) & =0, & & \forall v \in \mathbb{V}_{h} \\
b(u, q) & =0, & & \forall q \in \mathbb{Q}_{h}
\end{aligned}\right.
$$

Or, equivalently: Find $\tilde{u} \in \mathbb{T}_{h} \times \mathbb{V}_{h} \times \mathbb{Q}_{h}$ s. t.

$$
a_{\lambda}(\tilde{u})(\lambda, \xi)+j_{\lambda}(\lambda, \xi)+a_{u}(\tilde{u})(u, v)+b(v, p)-b(u, q)=l(\xi, v, q) \quad \forall \tilde{v} \in \mathbb{T}_{h} \times \mathbb{V}_{h} \times \mathbb{Q}_{h}
$$

where the stabilization term $j_{\lambda}(\cdot, \cdot)$ is given as follows

$$
j_{\lambda}(\lambda, \xi)=\sum_{E \in \mathcal{F}_{i}} \gamma_{\lambda}|E| \int_{E}[\nabla \lambda][\nabla \xi] d \sigma
$$

The stabilization (15) is consistent and makes the coercivity and continuity match in $\mathbb{T}_{h}$ associated with the norm $\|\cdot\| \cdot \|$, where

$$
\|\xi\|^{2}=\|\xi\|_{0}^{2}+j_{\lambda}(\xi, \xi)
$$




\section{Generalized discrete Newton}

We use the Newton method to approximate the nonlinear residuals. Let $\mathcal{R}(\tilde{u})=\left(\mathcal{R}_{\lambda}(\tilde{u}), \mathcal{R}_{u}(\tilde{u}), \mathcal{R}_{p}(\tilde{u})\right)=$ $\left(\mathcal{R}_{(\lambda, u)}(\tilde{u}), \mathcal{R}_{p}(\tilde{u})\right)$ denote the residuals for the system (14). The nonlinear iteration is updated with the correction $\delta \tilde{u}, \tilde{u}^{k+1}=\tilde{u}^{k}+\delta \tilde{u}$. Then, the Newton linearization gives the following approximation for the residuals:

$$
\begin{aligned}
\mathcal{R}\left(\tilde{u}^{l+1}\right) & =\mathcal{R}\left(\tilde{u}^{l}+\delta \tilde{u}\right) \\
& \simeq \mathcal{R}\left(\tilde{u}^{l}\right)+\left[\frac{\partial \mathcal{R}\left(\tilde{u}^{l}\right)}{\partial \tilde{u}}\right] \delta \tilde{u}
\end{aligned}
$$

The Newton's method iterations, assuming invertible Jacobians, are given as follows

$$
\tilde{u}^{l+1}=\tilde{u}^{l}-\omega_{l}\left[\frac{\partial \mathcal{R}\left(\tilde{u}^{l}\right)}{\partial \tilde{u}}\right]^{-1} \mathcal{R}\left(\tilde{u}^{l}\right)
$$

The damping parameter $\omega_{l} \in(0,1)$ is chosen such that

$$
\left\|\mathcal{R}\left(\tilde{u}^{l+1}\right)\right\| \leq\left\|\mathcal{R}\left(\tilde{u}^{l}\right)\right\|
$$

The damping parameter is not sufficient for the convergence of this type of highly nonlinear problem, mainly due to the presence of Jacobian's singularities related to the problem or simply by being out of the domain of Newton's convergence [5,6]. We use a generalized Newton's method which consists of using approximate Jaco

accurate Jacobians in the priori analysis of Jacobian
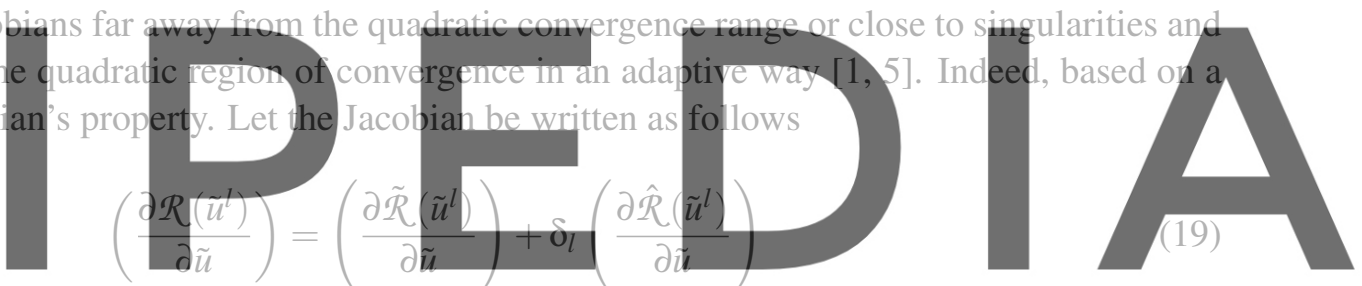

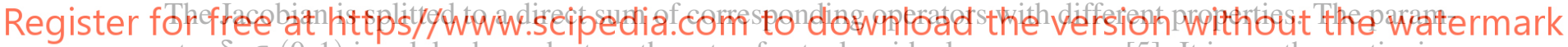
eter $\delta_{l} \in(0,1)$ is solely dependent on the rate of actual residual convergence [5]. It is worth mentioning that the operator-related damped Jacobian method (19) is related to the continuous Newton's method. Furthermore, for the analysis of adaptive discrete Newton's method where the Jacobian is evaluated using the divided difference we refer to [1]. The Jacobian approximation is only dependent on the rate of actual residual convergence $\left(\left\|R^{l}\right\| /\left\|R^{l-1}\right\|\right)$. This generalized Newton's method assures a global nonlinear convergence $[1,5]$.

\section{Monolithic multigrid linear solver}

To develop an appropriate linear solver, we segregate the Jacobian as follows

$$
\left(\frac{\partial \mathcal{R}(\tilde{u})}{\partial \tilde{u}}\right)=\left(\begin{array}{ll}
\frac{\partial \mathcal{R}_{(\lambda, u)}(\tilde{u})}{\partial(\lambda, u)} & \frac{\partial \mathcal{R}_{u}(\tilde{u})}{\partial p} \\
\frac{\partial \mathcal{R}_{p}(\tilde{u})}{\partial u} & 0
\end{array}\right)
$$


which is a saddle point problem. Then, the resulting linear system is treated with a Multilevel Pressure Schur Complement (MPSC) approach with Vanka-like smoother i.e.

$$
\tilde{u}^{k+1}=\tilde{u}^{k}-\omega_{k} \sum_{K \in \mathcal{T}_{h}}\left(\left(\frac{\partial \mathcal{R}\left(\tilde{u}^{l}\right)}{\partial \tilde{u}}\right)_{\mid K}\right)^{-1} \mathcal{R}\left(\tilde{u}^{l}\right)_{\mid K}
$$

In (21), we solve exactly on fixed element, $K$, and perform an outer Gauß-Seidel iteration $[2,11]$. We use standard geometric multigrid solver for linearized system with standard $Q_{2}$ and $P_{1}^{\text {disc }}$ restriction and prolongation operators.

The combination of a stable finite element approximations, $Q_{2} / P_{1}^{\text {disc }}$, for Stokes problem together with multigrid results in high numerically accurate, flexible, and efficient FEM-multigrid solver.

\section{Numerical Results and Discussions}

Our obiective is the investigation of thixotronic phenomena as a competition of two processes; build un and breakdown. Four continua models can be differentiated w.r.t. non-vanishing parameters: Newtonian $\left(\eta_{0} \neq 0\right)$, non-thixotropic $\left(\tau_{0} \neq 0\right)$, slightly-thixotropic $\left(\tau_{0} \neq 0, \tau_{1} \neq 0\right)$, and fully-thixotropic $\left(\tau_{1} \neq 0\right)$. For the complete analysis of thixotropic flows, a relative flow behavior is studied w.r.t. breakdown parameter $b$.

Prior to analyzing the complete problem, we consider two elementary test problems. On one hand, to validate our numerical schemes; on the other hand, to dive slowly into the problem complexities. We first simulate the fully developed flow of Bingham fluid. This test problem possesses an exact analytical solution, which is convenient for strdying the convergence of the computed solytion with mesh refinement. Our second test problem pertains to unidirectional flow of Houška's thixotropic material. Here w compare the computed so Finally, we examine the liquid-/solid transitions of thixotropy breakdown parameter for a model
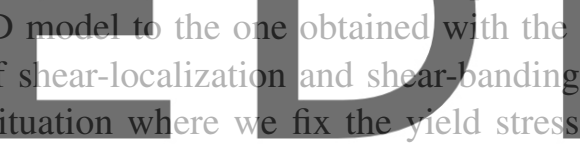

parameters $\left(\tau_{0}, \tau_{1}\right)$ and $\left(\eta_{0}, \eta_{1}\right)$ as well as the thixotropy buildup parameter $a$ in a Couette device.

Register for free at https//www.scipedia.com to download the version without the watermark

The analysis of our numerical methods for the channel flows is essential for its simplicity in terms of benchmarking and model calibrating. The numerical simulations are conducted for the manufactured exact solution for Bingham flow in a unit channel domain (22) where the flow is made under a constant pressure drop, $p_{\text {inflow }}-p_{\text {outflow }}=-1$, and boundaries conditions of the exact solution, $u_{\text {exact }}=(u, 0)^{\mathrm{T}}$, w.r.t. dimensionless $\tau_{0}$, s.t.

$$
u= \begin{cases}\frac{1}{8}\left(\left(1-2 \tau_{0}\right)^{2}-\left(1-2 \tau_{0}-2 y\right)^{2}\right) & \text { if } y \in\left[0, \frac{1}{2}-\tau_{0}\right) \\ \frac{1}{8}\left(1-2 \tau_{0}\right)^{2} & \text { if } y \in\left[\frac{1}{2}-\tau_{0}, \frac{1}{2}+\tau_{0}\right] \\ \frac{1}{8}\left(\left(1-2 \tau_{0}\right)^{2}-\left(2 y-2 \tau_{0}-1\right)^{2}\right) & \text { if } y \in\left(\frac{1}{2}+\tau_{0}, 1\right]\end{cases}
$$

The solution shows that the plug region, $y \in\left[\frac{1}{2}-\tau_{0}, \frac{1}{2}+\tau_{0}\right]$, is a moving rigid body with a constant velocity. We provide the numbers of Newton iterations in Table 1 , respectively the $L_{2}$-error of velocity in Table 2 for different values of $\tau_{0}$ and different mesh refinement. 
Table 1: Newton Iterations: Corresponding results of No. of non-linear iterations w.r.t. regularization parameter $k$ and refinement level $L$ for Bingham viscoplastic flow for different values of non-thixotropic yield stress parameter $\tau_{0}$.

\begin{tabular}{|c|c|c|c|c|c|c|c|c|c|c|}
\hline \multirow[t]{2}{*}{$k \backslash L$} & 2 & 3 & 4 & 5 & 6 & 2 & 3 & 4 & 5 & 6 \\
\hline & \multicolumn{5}{|c|}{ For $\tau_{0}=0.25$} & \multicolumn{5}{|c|}{ For $\tau_{0}=0.375$} \\
\hline $10^{1}$ & 3 & 3 & 4 & 3 & 4 & 3 & 3 & 3 & 3 & 3 \\
\hline $10^{2}$ & 15 & 15 & 14 & 12 & 12 & 11 & 11 & 11 & 11 & 11 \\
\hline $10^{3}$ & 16 & 17 & 18 & 16 & 16 & 13 & 17 & 17 & 16 & 19 \\
\hline $10^{4}$ & 22 & 22 & 24 & 24 & 25 & 15 & 23 & 25 & 26 & 26 \\
\hline $10^{5}$ & 28 & 22 & 27 & 29 & 32 & 11 & 25 & 29 & 32 & 35 \\
\hline $10^{6}$ & 34 & 22 & 30 & 50 & 52 & 9 & 25 & 32 & 51 & 53 \\
\hline
\end{tabular}

Table 2: $L^{2}$-error of velocity $\left\|u-u_{e x}\right\|_{2}$ : Velocity errors in $L_{2}$ for Bingham viscoplastic flow for different values of non-thixotropic yield stress.

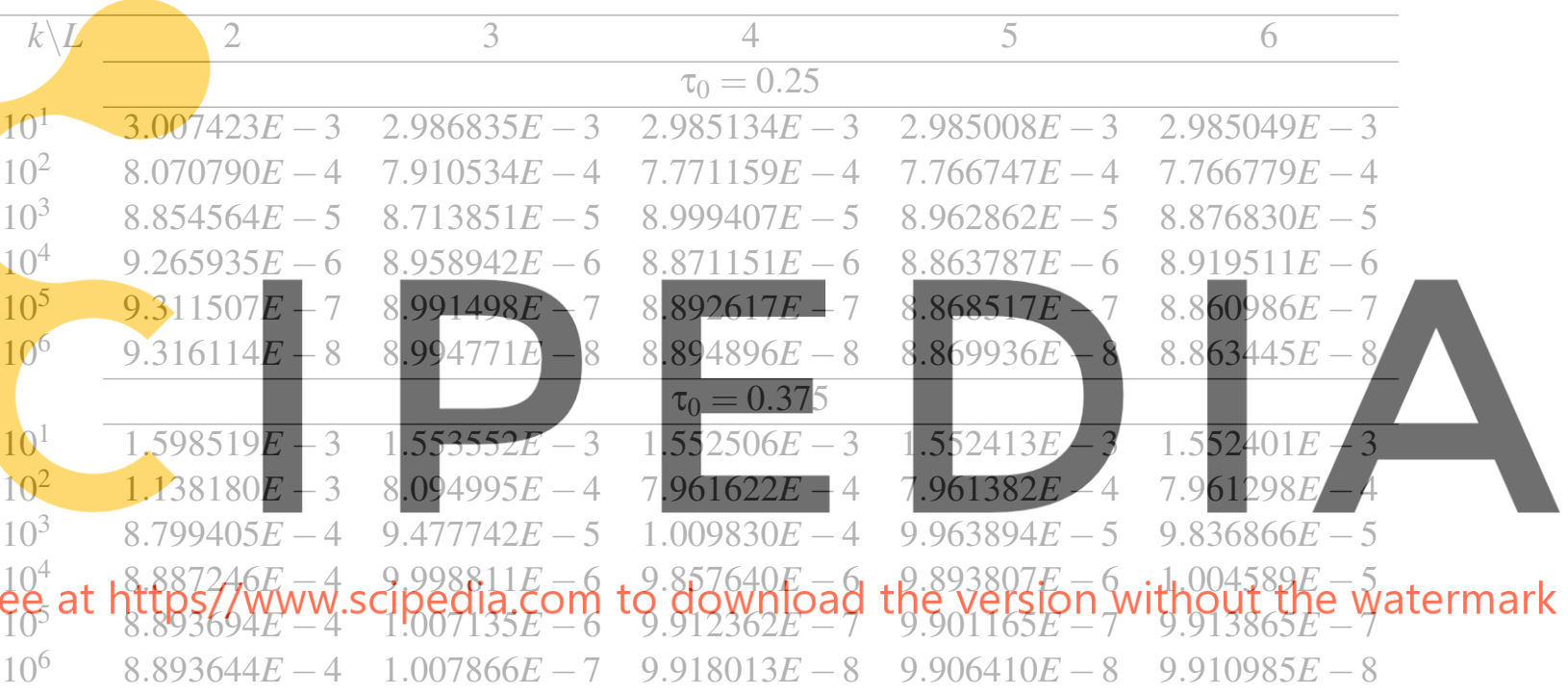

Clearly, the accuracy of the solution is dependent on the regularization parameters (see Table 2). Very high values for regularization are required to achieve accurate approximation. Fortunately, the solver is so robust that the solutions are obtained for higher values of the regularization parameter (see, Table 1). The lack of mesh convergence for the error in $L^{2}$-norm requires more theoretical investigations which go beyond the current work.

We examine the fully developed flow in a channel filled with a thixotropic material. In Figure 1, we present the evolution of material axial velocities and structure parameter computed with the simplified 1D model and analogize it to the predictions of the complete 2D model. From the curves being pretty much indistinguishable, we reinforce that the assumptions made in fully-developed thixotropic models are substantially satisfied. Our objective to do so is primarily to support the idea that, for a dominant viscous force, the final "spread" is only related to the yield stress of the material which plays a key for code validations. 

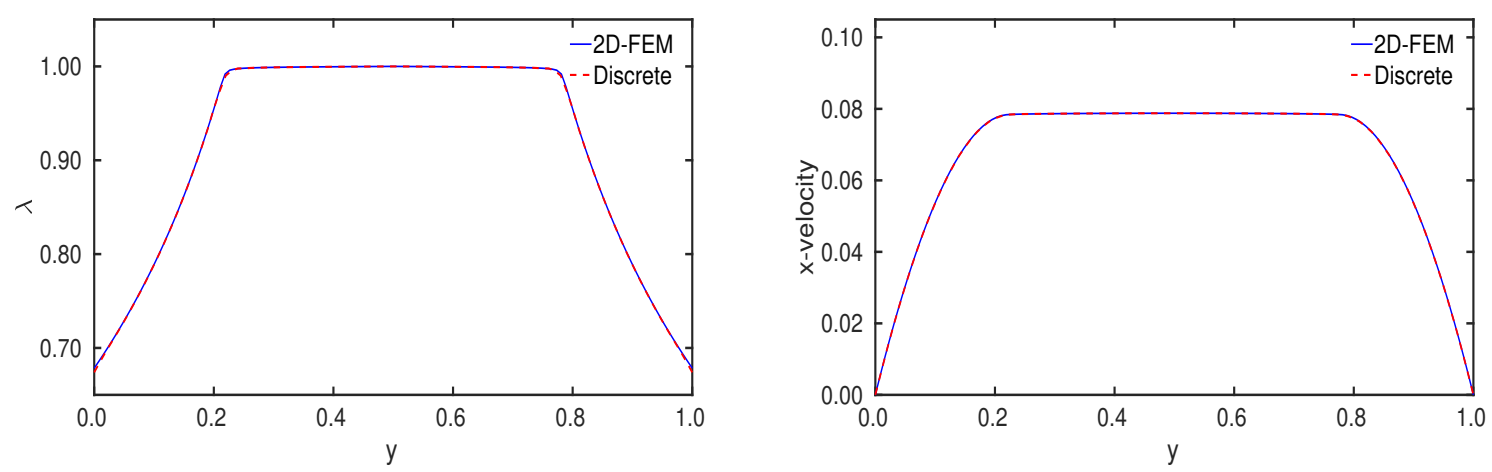

Figure 1: Thixotropic flows in in a channel: The comparison of 1D and a fully developed 2D models for thixotropic flows in a channel.

A detailed analysis of the complete thixotropic model in complex configuration of Couette type is illustrated in the next subsection.

\subsection{Thixotropic flows in Couette device}

For numerical solutions for thixotropic flows in Couette device, we consider two concentric cylinders with an inner and an outer radii $r_{\text {in }}$ and $r_{\text {out }}$ defined as $r_{\text {in }}=\zeta /(1-\zeta)$ and $r_{\text {out }}=1 /(1-\zeta)$; where $\zeta$ denotes the radii ratio. The inner cylinder is made under rotation speed, $u=u\left(r_{\text {in }}\right) e_{\theta} ; u\left(r_{\text {in }}\right)=1 \mathbb{R P M}$, while the outer cylinder is the pressure distribution, centric cylinders. From the results of velocity gr Eq.(1), are calculated for the
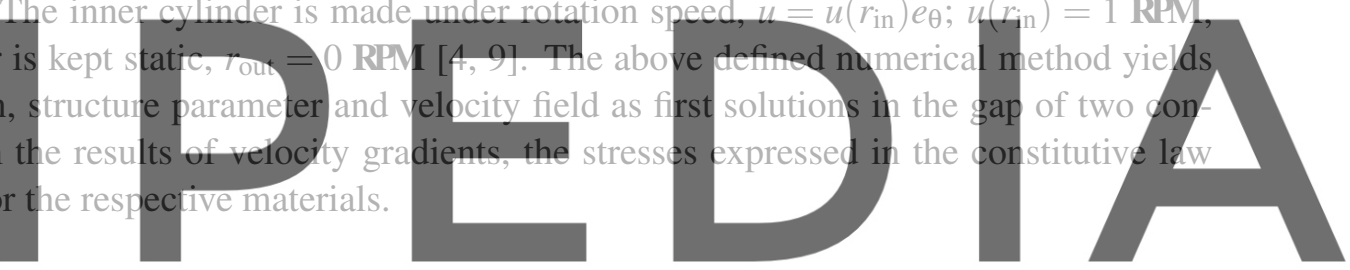

Using these results together with total stress tensor $\mathbf{T}_{i j}=-p \boldsymbol{I}+\sigma_{i j}$, the torque force, $F$, exerted on the

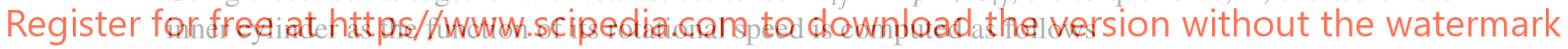

$$
F=-\oint_{S}\left(x-x_{0}\right) T_{i j} n d S
$$

where $S$ the inner-cylinder surface, $x_{0}$ the center of concentric Couette device and $n$ the directional vector.

Figure 2 depicts a sample calculation with the numerical method for the torque on the inner rotating cylinder as a function of the rotational speed of the inner cylinder (in RPM). Firstly, we validate our numerical method for Newtonian liquid and Bingham plastic w.r.t. literature [9]. Secondly, we show the four continua Newtonian liquid, Bingham plastic, Houška's slight thixotropic, and Houška's full thixotropic. The thixotropic flow is encompassed between the two extremities, Newtonian liquid and Bingham plastic which is a direct response of the model as linear combination with a structure parameter. In the next numerical simulations, we investigate the spatial transitions in thixotropic flows.

We show the radial solutions profiles, velocity, structure parameter, and shear rate w.r.t. breakdown parameters.

Figure 3 shows the flow characteristics w.r.t. breakdown parameter. A smooth transition from the flowing 

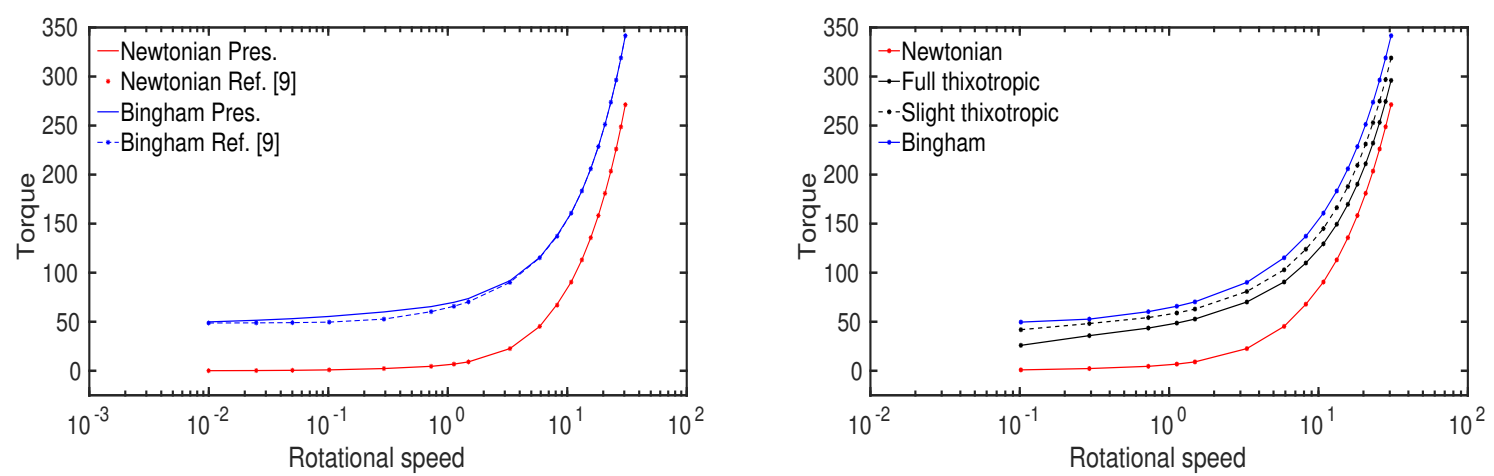

Figure 2: Thixotropic flows in Couette device: The torque on the inner rotating cylinder as a function of the rotational speed of the inner cylinder (in RPM). Validation w.r.t. literature [9] for (a) Newtonian fluid, (b) Bingham plastic materials (LEFT). The full spectrum of the numerical solution of thixotropic flows (a) Newtonian fluid, (b) Bingham plastic, (c) Houška's slight thixotropic (d) Houška's full thixotropic (RIGHT).

regimes to static ones is given with a small values of the breakdown parameter. The model allows for very sharp transitions as well for higher breakdown parameter. The structure parameter which control the buildup and the breakdown regions matches perfectly with the flowing and plug zones indicated by the shear rate. Furthermore, the velocity on one hand and the shear rate and structure parameter on the other hand come into contact perfectly in the transition points for both "localization" (lower breakdown

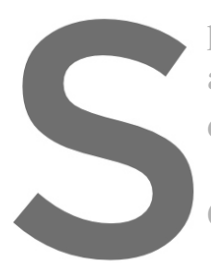
parameter, $b=0.5$ ) an a discontinuity jump to in diverging as for a simple y 6 Summary
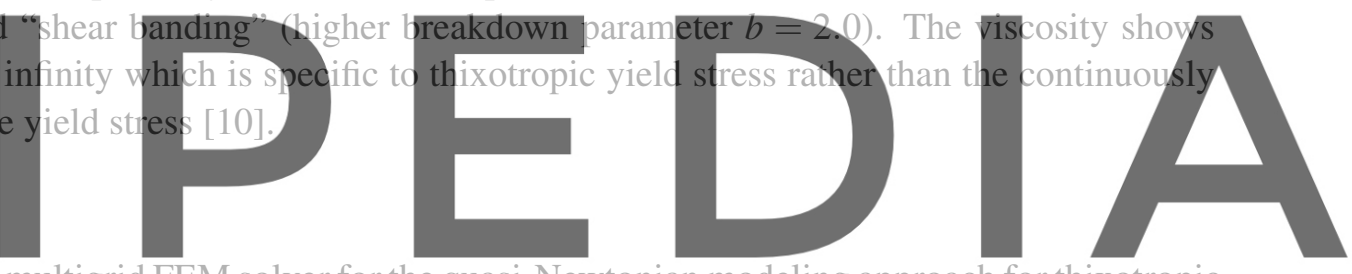

We presented a Newton-multigrid FEM solver for the quasi-Newtonian modeling approach for thixotropic

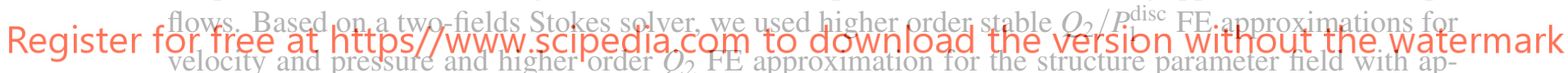
propriate stabilization term. The combination of a stable finite element approximations, $Q_{2} / P_{1}^{\text {disc }}$, for

Stokes problem together with multigrid results in high numerically accurate, flexible and efficient FEMmultigrid solver.

The quasi-Newtonian model for thixotropic flows is highly nonlinear due to the dependency of the extended viscosity on the material parameters (shear rate and structure-parameter) and the regularization parameter. The nonlinearity is handled with generalized Newton's method w.r.t. the Jacobian's singularities having a global convergence property.

We analyzed the application of using the quasi-Newtonian modeling approach for thixotropic flows, and the accuracy, robustness and efficiency of the Newton-Multigrid FEM solver throughout the solution of the thixotropic flows using manufactured solutions in a channel and the prototypical configuration of thixotropic flows in Couette device.

Very high values for regularization are required to achieve accurate solutions. Fortunately, the solver is so robust that the solutions are obtained for higher values for regularization parameter. Consequently, some 

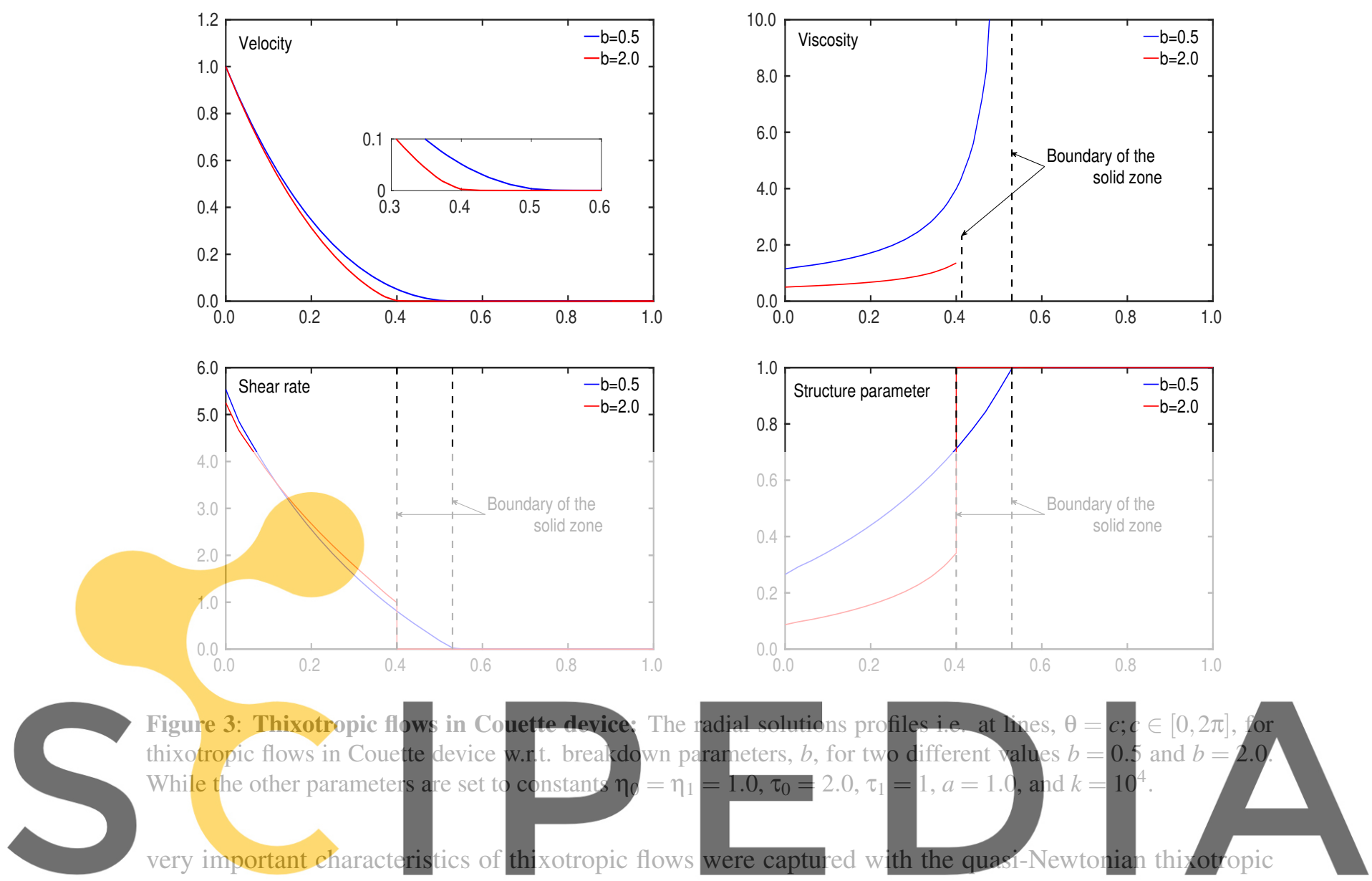

model; as for instance, localization, shear banding, and discontinuous jump to infinity for viscosity.

Register for free at https//www.scipedia.com to download the version without the watermark

[1] Fatima, A., Turek, S. and Ouazzi, A. An adaptive discrete Newton method for regularization-free Bingham model. Ergebnisberichte des Instituts für Angewandte Mathematik Nummer 635, Fakultät für Mathematik, TU Dortmund University 635, (2021)

[2] Damanik, H. and Hron, J. and Ouazzi, A. and Turek, S. A monolithic FEM-multigrid solver for non-isothermal incompressible flow on general meshes. J. Comp. Phys. (2009) 228:3869-3881.

[3] Houška, M. Engineering aspects of the rheology of thixotropic liquids. PhD thesis, Faculty of Mechanical Engineering, Czech Technical University of Prague, (1981).

[4] Jenny, M. and Kiesgen de Richter, S. and Louvet, N. and Skali-Lami, S. and Dossmann, Y. TaylorCouette instability in thixotropic yield stress fluids. Phys. Rev. Fluids (2017) 2:023302-023323.

[5] Mandal, S. and Ouazzi, A. and Turek, S. Modified Newton solver for yield stress fluids, Proceedings of ENUMATH 2015, the 11th European Conference on Numerical Mathematics and Advanced Applications, Springer,(2016):481-490 
[6] Ouazzi, A. Finite Element Simulation of Nonlinear Fluids. Application to Granular Material and Powder, Shaker Verlag, Achen (2006).

[7] Turek, S. and Ouazzi, A. Unified edge-oriented stabilization of nonconforming FEM for incompressible flow problems: Numerical investigations. Journal of Numerical Mathematics (2007) 15(4):299-322.

[8] Papanastasiuo, T.C., Flow of materials with yield. J. Rheol., (1987) 31:385-404.

[9] Kheiripour Langroudi, M. and Turek, S. and Ouazzi, A. and Tardos, G., An investigation of frictional and collisional powder flows using a unified constitutive equation. Powder Technology, (2009) 197(1-2):91-101.

[10] Moller, P. and Fall, A. and Chikkadi, V. and Derks, D. and Bonn, D, An attempt to categorize yield stress fluid behaviour. Phil Trans. R. Soc. A, (2009) 367:5139-5155.

[11] Turek, S. Efficient solvers for incompressible flow problems. Lecture Notes in Computational Science and Engineering, Springer, Berlin (1999).
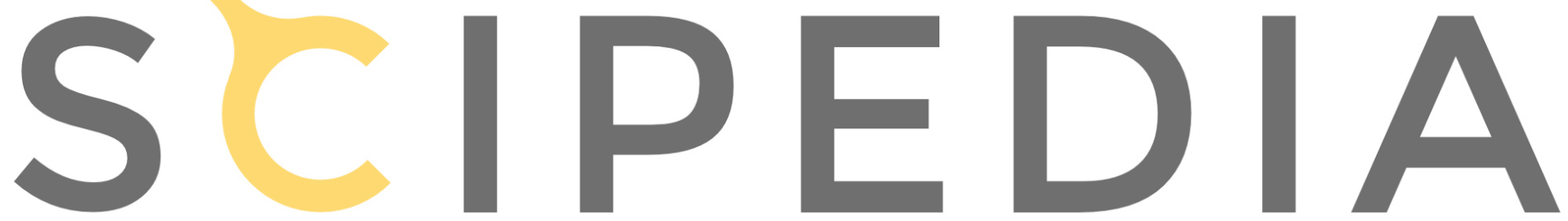